\title{
25 Research Square \\ Radiographic Evaluation of Congruency of the First Metatarsophalangeal Joint in Hallux Valgus
}

\section{Yan Li}

Southwest Hospital

Xu Tao ( $\square$ taoux@hotmail.com )

Southwest Hospital https://orcid.org/0000-0001-6533-9927

Kanglai Tang

Southwest Hospital

\section{Research article}

Keywords: Hallux valgus, Metatarsophalangeal joint, Congruency, Metatarsophalangeal joint angle, Congruency index

Posted Date: November 1st, 2021

DOI: https://doi.org/10.21203/rs.3.rs-965019/v1

License: (9) (1) This work is licensed under a Creative Commons Attribution 4.0 International License. Read Full License 


\section{Abstract}

Background Congruency of the first metatarsophalangeal (MTP) joint is extremely important for the selection of surgical methods and prognosis, while radiographic evaluation methods are relatively lacking. The purpose of this article was to explore radiographic indicators for evaluating congruency of the first MTP joint.

Methods Patients who had a weightbearing X-ray in the outpatient system were selected, excluding patients with trauma, deformity, and history of surgery. A total of 183 patients with 245 feet were included. The hallux valgus angle (HVA), distal metatarsal articular angle (DMAA), metatarsophalangeal joint angle (MTPJA), congruency index $(\mathrm{Cl})$ and tibial sesamoid position (TSP) were measured and statistically analysed.

Results The higher the degree of hallux valgus was, the higher the proportion of incongruency of the first MTP joint. Significant differences were found in the DMAA, MTPJA and $\mathrm{Cl}$ between the congruency and incongruency groups of patients with moderate to severe hallux valgus $(P<0.05)$. The areas under the curve (AUCs) of the receiver operating characteristic (ROC) curve for the MTPJA and $\mathrm{Cl}$ were 0.906 and 0.884 , the sensitivity values reached 0.791 and 0.949 , the specificity values were 0.862 and 0.644 , and the critical values were 10.67 and 0.765 , respectively. The correlation test indicated that in the congruency group, the DMAA and HVA were positively correlated, but the MTPJA, $\mathrm{Cl}$ and HVA had low correlation coefficients. The DMAA and HVA were not correlated in the incongruency group, while the MTPJA and HVA were significantly positively correlated and the $\mathrm{Cl}$ and $\mathrm{HVA}$ showed a negative correlation $(P<0.05)$.

Conclusion The MTPJA and $\mathrm{Cl}$ are indicators that can be used to quantitatively evaluate the congruency of the first MTP joint, taking $10^{\circ}$ and 0.765 as the demarcation points, respectively. Clinically, congruency of the MTP joint should be considered when choosing surgical methods for different degrees of hallux valgus, and the MTPJA and $\mathrm{Cl}$ can be used as quantitative evaluation indicators.

Level of evidence: Level III, Retrospective Comparative Study

\section{Background}

Congruency of the first metatarsophalangeal (MTP) joint is important for the choice of hallux valgus surgery and postoperative recurrence ${ }^{[1]}$. However, the data on the congruency of the MTP joint are quite different ${ }^{[2,3]}$. Patients with moderate to severe hallux valgus have a high degree of congruency and incongruency of the MTP joint. For patients with hallux valgus of the same degree, there are large differences in the choice of surgical methods due to the existence of MTP joint congruency and incongruency. Double metatarsal osteotomy (DMO) is often required for patients who have a large hallux valgus angle (HVA) and congruency of the MTP joint ${ }^{[4,5]}$. The congruency of the first MTP joint is calculated with two lines that refer to the base of the proximal phalanx and the estimated cartilage surface of the first metatarsal head, although there is no quantitative measurement index for the congruency of the first MTP joint ${ }^{[8]}$.

The purpose of this article was to quantitatively evaluate the congruency of the MTP joint through two innovative indicators and to conduct a diagnostic experimental analysis, aiming to assess the evaluation value of the two new indicators and the intrinsic relationship between different MTP joint radiographic indicators. This study also provides a basis for the selection of surgical options for patients with different types of hallux valgus and the prevention of postoperative recurrence. 


\section{Methods}

Patients with hallux valgus who had a weightbearing X-ray in the outpatient system from January 2018 to January 2021 were selected. Patients with ankle trauma, fractures, and a history of previous surgeries or those younger than 18 years old were excluded. Finally, 183 hallux valgus patients with 245 feet were included, comprising 51 males and 132 females. Among the patients, 131 feet (53.47\%) had mild hallux valgus (HVA: 15$30^{\circ}$ ), 80 feet $(32.65 \%)$ had moderate hallux valgus (HVA: $\left.31-40^{\circ}\right)$, and 34 feet $(13.88 \%)$ had severe hallux valgus $\left(\mathrm{HVA} \geq 40^{\circ}\right)$ according to the classification criteria for hallux valgus.

The HVA, distal metatarsal articular angle (DMAA), and tibial sesamoid position (TSP) were measured in weightbearing foot anterior-posterior images according to the measurement method described in previous literature (Fig. 1A, B, E) ${ }^{[6]}$. According to the congruency and incongruency of the first MTP joint in patients with hallux valgus, we innovatively designed two new measurement indicators, the metatarsophalangeal joint angle (MTPJA) and congruency index (CI), which was defined by the MTPJA as follows: on the weightbearing foot anterior-posterior images, we drew a straight line between the inner and outer edges of the articular surface of the proximal phalanx and the distal metatarsal, respectively, and the angle between the two straight lines was the MTPJA (Fig. 1C). The $\mathrm{Cl}$ was calculated as described in our previous article ${ }^{[7]}$. The ratio of the curve length of the metatarsophalangeal joint contact surface to the curve length of the metatarsal head cartilage surface was defined as the $\mathrm{Cl}$, which was designed to measure the congruency of the MTP joint (Fig. 1D). There are currently no quantitative indicators to assess whether the metatarsophalangeal joint is congruent; it is based only on the physician's subjective observation of whether the articular surfaces at both ends of the metatarsophalangeal joint were parallel (Fig. 1F). Based on this, we selected two doctors with 20 years of experience in foot and ankle surgery and divided the 245 feet into the congruency and incongruency groups. The HVA, DMAA, MTPJA, CI and TSP were statistically analysed among different degrees of hallux valgus and between the congruency and incongruency groups. Diagnostic tests were performed for the MTPJA and $\mathrm{Cl}$, and receiver operating characteristic (ROC) curves were plotted. The area under the curve (AUC), sensitivity, specificity and critical value were calculated.

SPSS 20.0 software was used for statistical analysis. Quantitative data were expressed as X \pm S. The comparison of different parameters between the congruency and incongruency groups of patients with different degrees of hallux valgus was performed with independent sample Student's $t$ test. The diagnostic test of the MTPJA and CI used ROC curves and calculated the AUC, critical value, sensitivity, specificity and other parameters. A correlation test was carried out between different parameters of the congruency and incongruency groups, and the correlation coefficient was calculated. The difference was considered to be statistically significant at $\mathrm{P}<0.05$.

\section{Results}

The HVA, DMAA, MTPJA and TSP increased significantly as the degree of hallux valgus increased, while the Cl decreased $(P<0.05)$ (Table 1). Using the previous observation of the parallelism of the first metatarsophalangeal joint surface as the gold standard ${ }^{[8]}$, we divided 245 feet into the congruency and incongruency groups. In total, approximately $2 / 3(64.49 \%)$ of the patients had first MTP joints that were congruent, and approximately $1 / 3$ $(35.51 \%)$ of the patients had first MTP joints that were incongruent. The majority of patients with mild hallux valgus have congruency, while those with severe hallux valgus have incongruency. Patients with moderate hallux valgus were basically the same $(P<0.05)$ (Fig. 2). 
Table 1

Comparison and analysis of different indexes in patients with different degrees of hallux valgus.

\begin{tabular}{|c|c|c|c|c|c|c|}
\hline Index & $\begin{array}{l}\text { Mild hallux } \\
\text { valgus }\end{array}$ & $\mathbf{P}^{*}$ & $\begin{array}{l}\text { Moderate hallux } \\
\text { valgus }\end{array}$ & $\mathrm{P}^{* \star}$ & $\begin{array}{l}\text { Severe hallux } \\
\text { valgus }\end{array}$ & $P^{* \star *}$ \\
\hline $\operatorname{Sex}(M / F)$ & $37 / 70$ & 0.060 & $15 / 55$ & 0.637 & $5 / 24$ & 0.073 \\
\hline Age (year) & $45.60 \pm 16.55$ & 0.064 & $51.44 \pm 17.33$ & 0.116 & $57.34 \pm 17.21$ & 0.003 \\
\hline L/R & $69 / 62$ & 0.981 & $42 / 38$ & 0.807 & $17 / 17$ & 0.781 \\
\hline $\mathrm{HVA}\left({ }^{\circ}\right)$ & $24.07 \pm 4.01$ & 0.000 & $33.72 \pm 2.79$ & 0.000 & $46.52 \pm 4.71$ & 0.000 \\
\hline $\operatorname{DMAA}\left({ }^{\circ}\right)$ & $12.55 \pm 5.78$ & 0.000 & $18.98 \pm 7.26$ & 0.040 & $22.01 \pm 10.75$ & 0.000 \\
\hline $\operatorname{MTPJA}\left({ }^{\circ}\right)$ & $8.25 \pm 4.88$ & 0.000 & $12.91 \pm 7.45$ & 0.000 & $23.42 \pm 13.90$ & 0.000 \\
\hline $\mathrm{Cl}$ & $0.85 \pm 0.07$ & 0.000 & $0.80 \pm 0.09$ & 0.000 & $0.65 \pm 0.16$ & 0.000 \\
\hline TSP & $3.87 \pm 1.65$ & 0.000 & $5.15 \pm 1.67$ & 0.004 & $6.09 \pm 1.08$ & 0.000 \\
\hline Feet number (ratio) & $131(53.47 \%)$ & 0.003 & $80(32.65 \%)$ & 0.000 & $34(13.88 \%)$ & 0.000 \\
\hline Congruency/Incongruency & $112 / 19$ & 0.000 & $38 / 42$ & 0.017 & $8 / 26$ & 0.000 \\
\hline \multicolumn{7}{|c|}{$\begin{array}{l}\text { Abbreviations: HVA, Hallux valgus angle; DMAA, Distal metatarsal articular angle; MTPJA, The first } \\
\text { metatarsophalangeal joint angle; Cl, Congruency index; TSP, Tibial sesamoid position }\end{array}$} \\
\hline
\end{tabular}

Although the difference in HVA between the two groups of patients with mild and moderate hallux valgus was statistically significant, the numerical differences were not significant. Patients with severe hallux valgus did not have a significant difference in HVA between the two groups. In the congruency group, the TSP of patients with mild to moderate hallux valgus was lower than that of the incongruency group, while there was no significant difference between the two groups of patients with severe hallux valgus. In terms of DMAA, the mild patients had no significant difference between the congruency and incongruency groups, but for moderate to severe hallux valgus, the DMAA of the congruency group was greater than that of the incongruency group. Among all patients, the MTPJA was lower, and the $\mathrm{Cl}$ was greater in the congruency group than in the incongruency group (Table 2). 
Table 2

Comparison and analysis of different index of patients with different degrees of hallux valgus between congruency and incongruency groups

\begin{tabular}{|c|c|c|c|c|c|}
\hline Groups & Index & Congruency & Incongruency & $\mathrm{T}$ value & $P$ value \\
\hline \multirow[t]{5}{*}{ Mild } & $\operatorname{HVA}\left({ }^{\circ}\right)$ & $23.60 \pm 4.00$ & $26.78 \pm 2.88$ & -4.172 & 0.000 \\
\hline & $\operatorname{MTPJA}\left({ }^{\circ}\right)$ & $7.41 \pm 3.99$ & $13.23 \pm 6.58$ & -3.744 & 0.001 \\
\hline & $\operatorname{DMAA}\left({ }^{\circ}\right)$ & $11.27 \pm 5.69$ & $14.20 \pm 6.22$ & -1.350 & 0.179 \\
\hline & $\mathrm{Cl}$ & $0.86 \pm 0.06$ & $0.78 \pm 0.07$ & 5.071 & 0.000 \\
\hline & TSP & $3.55 \pm 1.55$ & $5.74 \pm 0.81$ & -9.265 & 0.000 \\
\hline \multirow[t]{5}{*}{ Moderate } & $\operatorname{HVA}\left({ }^{\circ}\right)$ & $32.98 \pm 2.54$ & $34.39 \pm 2.86$ & -2.329 & 0.022 \\
\hline & $\operatorname{MTPJA}\left({ }^{\circ}\right)$ & $7.57 \pm 4.72$ & $17.73 \pm 6.05$ & -8.309 & 0.000 \\
\hline & $\operatorname{DMAA}\left({ }^{\circ}\right)$ & $22.04 \pm 7.77$ & $16.22 \pm 5.54$ & 3.888 & 0.000 \\
\hline & $\mathrm{Cl}$ & $0.85 \pm 0.06$ & $0.75 \pm 0.08$ & 6.932 & 0.000 \\
\hline & TSP & $4.37 \pm 1.85$ & $5.86 \pm 1.09$ & -4.320 & 0.000 \\
\hline \multirow[t]{5}{*}{ Severe } & $\operatorname{HVA}\left({ }^{\circ}\right)$ & $43.98 \pm 1.89$ & $47.30 \pm 5.06$ & -1.803 & 0.081 \\
\hline & $\operatorname{MTPJA}\left({ }^{\circ}\right)$ & $4.90 \pm 3.32$ & $29.12 \pm 10.42$ & -6.415 & 0.000 \\
\hline & $\operatorname{DMAA}\left({ }^{\circ}\right)$ & $32.45 \pm 5.04$ & $18.79 \pm 10.00$ & 5.154 & 0.000 \\
\hline & $\mathrm{Cl}$ & $0.80 \pm 0.05$ & $0.61 \pm 0.15$ & 5.925 & 0.000 \\
\hline & TSP & $5.50 \pm 1.41$ & $6.27 \pm 0.92$ & -1.447 & 0.182 \\
\hline
\end{tabular}

Because of the significant difference in the MTPJA and $\mathrm{Cl}$ between the two groups of patients with hallux valgus of different degrees $(\mathrm{P}<0.001)$, we performed diagnostic tests and plotted the ROC curve with the MTPJA and CI data. The AUCs of the MTPJA and CI were 0.906 and 0.884 , respectively, which were both greater than 0.7 $(P<0.001)$, and showed significant diagnostic value (Figs. 3\&4). The sensitivity and specificity of the MTPJA reached 0.791 and 0.862 , respectively, and the sensitivity and specificity of the $\mathrm{Cl}$ reached 0.949 and 0.644 . In addition, the critical value of the MTPJA was 10.67, and that of the $\mathrm{Cl}$ was 0.765 (Table 3 ). 
Table 3

Diagnostic test between two new index and congruency of the metatarsophalangeal joint

\begin{tabular}{|llllllllllll}
\hline Index & $\begin{array}{l}\text { Critical } \\
\text { value }\end{array}$ & Sensitivity & Specificity & AUC & $\begin{array}{l}\text { P } \\
\text { value }\end{array}$ & $\begin{array}{l}\text { Youden } \\
\text { index }\end{array}$ & PPV & NPV & +LR & -LR \\
\hline MTPJA & 10.670 & 0.791 & 0.862 & 0.906 & 0.000 & 0.653 & 0.912 & 0.694 & 5.736 & 0.242 \\
$\mathrm{Cl}$ & 0.765 & 0.949 & 0.644 & 0.884 & 0.000 & 0.593 & 0.829 & 0.875 & 2.664 & 0.079 \\
\hline
\end{tabular}

Abbreviations: MTPJA, The first metatarsophalangeal joint angle; Cl, Congruency index; AUC, Area under the Curve; PPV, Positive Predictive Value; NPV, Negative predictive value; +LR, Positive likelihood ratio; -LR, Negative likelihood ratio

Correlation tests were performed on 5 parameters between the two groups of patients. In the congruency group, the DMAA was positively correlated with the HVA, while the correlation coefficients between the MTPJA, CI and HVA were low. In the incongruency group, the DMAA was not correlated with the HVA, while the MTPJA was positively correlated with the HVA, and the CI was negatively correlated with the HVA. The MTPJA and CI were negatively correlated in both the congruency group and the incongruency group (Tables $4 \& 5$ ).

Table 4

Correlation test of different index in the congruency group

\begin{tabular}{|lllll|}
\hline Index & MTPJA & DMAA & Cl & TSP \\
\hline HVA & $0.103(0.197)$ & $0.691(0.000)$ & $-0.272(0.001)$ & $0.345(0.000)$ \\
\hline MTPJA & - & $-0.377(0.000)$ & $-0.538(0.000)$ & $0.036(0.654)$ \\
\hline DMAA & - & - & $0.057(0.475)$ & $0.517(0.000)$ \\
\hline Cl & - & - & $-0.392(0.000)$ \\
\hline $\begin{array}{l}\text { Abbreviations: HVA, Hallux valgus angle; DMAA, Distal metatarsal articular angle; MTPJA, The first } \\
\text { metatarsophalangeal joint angle; Cl, Congruency index; TSP, Tibial sesamoid position }\end{array}$
\end{tabular}

Table 5

Correlation test of different index in the incongruency group

\begin{tabular}{|lllll|}
\hline Index & MTPJA & DMAA & Cl & TSP \\
\hline HVA & $0.554(0.000)$ & $0.212(0.048)$ & $-0.546(0.000)$ & $0.331(0.002)$ \\
\hline MTPJA & - & $-0.468(0.000)$ & $-0.760(0.000)$ & $0.389(0.000)$ \\
\hline DMAA & - & - & $0.322(0.002)$ & $-0.101(0.350)$ \\
Cl & - & - & - & $-0.557(0.000)$ \\
\hline
\end{tabular}

Abbreviations: HVA, Hallux valgus angle; DMAA, Distal metatarsal articular angle; MTPJA, The first metatarsophalangeal joint angle; Cl, Congruency index; TSP, Tibial sesamoid position

\section{Discussion}


The congruency of the first MTP joint plays an important role in the selection of the surgical method for postoperative recurrence in hallux valgus. Studies have shown that incongruency of the postoperative MTP joint was highly correlated with the recurrence of hallux valgus ${ }^{[8]}$. Previous literature reported ${ }^{[8]}$ that the congruency of the first MTP joint has been evaluated only by a doctor's visual assessment of whether the arcs of the MTP joint articular surface were parallel; however, there is no quantitative indicator. In addition, the DMAA is often used to assess dislocation of the first MTP joint ${ }^{[9]}$. For example, for patients with severe hallux valgus accompanied by an increased DMAA, double metatarsal osteotomy (DMO) is an effective surgical method ${ }^{[10]}$. However, due to the related complications, such as shortening the length of the first metatarsal bone, postoperative avascular necrosis of the metatarsal head, and metastatic metatarsalgia, the application of this technique has been limited to a certain extent ${ }^{[11]}$. Wang's study compared the efficacy of rotating scarf osteotomy and DMO for hallux valgus accompanied by an increased DMAA. It was believed that there was no significant difference between the two methods, but the former had a lower incidence of complications ${ }^{[11]}$. Jeong used the point-connecting method to measure the HVA and intermetatarsal angle (IMA), also considering the congruency and incongruency of the MTP joints, which would affect the assessment of the severity of hallux valgus ${ }^{[12]}$. supported

Evidence has supported that the DMAA is not suitable for use in evaluating the congruency of the first MTP joint ${ }^{[13]}$. For the larger metatarsals of the DMAA, there will also be two situations where the MTP joints are congruent and incongruent. The statistical results showed that there was an obvious relationship between the patients' DMAA and the congruency of the first MTP joint for moderate to severe hallux valgus. The DMAA of the congruency group was significantly larger than that of the incongruency group, indicating that the application of the DMAA alone to assess whether the first MTP joint was congruent was not reliable. We innovatively proposed two quantitative evaluation indexes for congruency of the first MTP joint on weightbearing foot anterior-posterior images, the MTPJA and $\mathrm{Cl}$, which were quantitatively assessed by measuring the angles of the articular surfaces at both ends and the degree of bonding of the articular surfaces (Fig. 1). Because our data were not clinically necessary, many asymptomatic patients were also included, resulting in a larger proportion of patients with mild hallux valgus, most of whom had congruent MTP joints. The proportions of patients with congruency and incongruency with moderate hallux valgus was basically the same. For patients with severe hallux valgus, nearly a quarter of them had congruency of the first MTP joint, which is similar to the data reported by Coughlin ${ }^{[14]}$. In addition, as the severity of hallux valgus gradually increased, the MTPJA gradually increased, and the $\mathrm{Cl}$ gradually decreased, indicating that the contact surface of the first MTP joint surface will gradually decrease.

In comparing the difference between the congruency and incongruency groups, there was no difference in the HVA between the two groups of patients with mild, moderate or severe hallux valgus. However, among patients with moderate to severe hallux valgus, the difference between the MTPJA and $\mathrm{Cl}$ was large; moreover, the $\mathrm{Cl}$ of the congruency group was greater than that of the incongruency group, and the MTPJA of the congruency group was smaller than that of the incongruency group, indicating that the MTPJA and Cl could be used to effectively assess the congruency of the first MTP joint. ROC curves of the MTPJA and Cl showed that the AUCs were 0.906 and 0.884 , respectively, and that both had diagnostic power. The critical value of the MTPJA was 10.67 , and that of the $\mathrm{Cl}$ was 0.765 . We can thus define the first MTP joint as incongruent if the value of MTPJA is greater than $10^{\circ}$ or $\mathrm{Cl}$ is less than 0.765 , and the degree of incongruency can be measured by the specific value of the two. That is, the larger the MTPJA and the smaller the $\mathrm{Cl}$, the greater the degree of congruency is. In our previous article $\mathrm{e}^{[7]}, 36$ patients (38 feet) with moderate to severe hallux valgus were followed up at different times before and after 
surgery, and their $\mathrm{Cl}$ recovered from 0.75 before surgery to 0.95 at the last follow-up. Similar to the HVA, IMA, etc., all of the indicators recovered well, which also verifies the effectiveness of this indicator to a certain extent.

In terms of the correlation test, the DMAA and HVA were positively correlated in the congruency group, while there was no correlation between the two in the incongruency group. This indicates that if the DMAA is to be used to assess the degree of hallux valgus, it is limited to the congruency group. In patients with significant dislocation of the first MTP joint, the DMAA is less effective in assessing the severity of hallux valgus. Therefore, for more severe hallux valgus surgery, the DMAA still needs to be measured in the congruency group, and the value of the DMAA will be used to determine whether to perform DMO. In the congruency group, the correlation coefficients between the MTPJA, Cl and HVA were low, while in the incongruency group, the MTPJA and HVA were significantly positively correlated and the $\mathrm{Cl}$ and HVA were negatively correlated; that is, the more severe the hallux valgus is, the more deviated the normal ranges of the MTPJA and $\mathrm{Cl}$ are. Of course, the smaller the value of the MTPJA and the larger the value of the $\mathrm{Cl}$, the better the matching relationship of hallux valgus is. Therefore, whether in the congruency group or incongruency group, there was a significant negative correlation between the two.

The present paper also has some limitations that should be taken into consideration. First, this study focused only on the statistical analysis of radiological parameters and did not apply the MTPJA and CI to the comparison of parameters before and after the operation in hallux valgus patients. Nor did it classify the magnitude of the two parameters relative to the clinical symptoms. This is what we need to include in the next step of our research. In addition, the patients included in this study had a certain deviation. The number of patients with mild hallux valgus was too large, but because metatarsophalangeal joint mismatch mostly occurs in patients with moderate to severe hallux valgus, we believe that the data in the study are still reliable.

In summary, in hallux valgus of different degrees, especially in patients with moderate to severe hallux valgus, the first MTP joint is either congruent or incongruent. The DMAA has poor performance in evaluating matching relationships, and the previously used imaging indicators are only qualitative evaluations. The MTPJA and CI can be used to quantitatively evaluate the congruency of the first MTP joint, and $10^{\circ}$ and 0.765 are used as the demarcation points. Clinically, it is necessary to consider the congruency of the first MTP joint in the selection of different degrees of hallux valgus surgery. The MTPJA and $\mathrm{Cl}$ can be used as quantitative evaluation indicators. That is, for patients with small MTPJA or large Cl, Scarf or Chevron osteotomy can be performed alone. For patients with MTPJA far greater than $10^{\circ}$ or $\mathrm{Cl}$ far less than 0.765 , it is necessary to consider whether to perform double metatarsal osteotomy to correct DMAA.

\section{Declarations}

\section{Ethics approval}

This study was approved by the Ethics Committee, and the part number was: (B) KY2021052.

\section{Consent for publication}

Not applicable.

\section{Availability of data and materials}

The datasets used during the current study are available from the corresponding author on reasonable request. 


\section{Conflicts of interest}

None of the authors have any conflicts of interest to declare.

\section{Funding}

Leading Scientific and Technological Innovation Talents Under the National“Ten Thousand People Plan"(4139Z2B1).

\section{Authors' contributions}

Yan Li was in charge of manuscript writing, clinical data collection and data statistics; Xu Tao and Kanglai Tang were in charge of the overall design and acceptance.

\section{Acknowledgments}

Not applicable.

\section{References}

1. Castioni D, Fanelli D, Gasparini G, et al. Scarf osteotomy for the treatment of moderate to severe hallux valgus: Analysis of predictors for midterm outcomes and recurrence[J]. Foot and Ankle Surgery, 2020,26(4):439-444. DOI: 10.1016/j.fas.2019.05.013.

2. Pentikainen I, Ojala R, Ohtonen P, et al. Preoperative Radiological Factors Correlated to Long-Term Recurrence of Hallux Valgus Following Distal Chevron Osteotomy[J]. Foot \& Ankle International, 2014,35(12):1262-1267. DOI: $10.1177 / 1071100714548703$.

3. Deveci A, Firat A, Yilmaz S, et al. Short-term clinical and radiologic results of the scarf osteotomy: what factors contribute to recurrence?[J]. J Foot Ankle Surg, 2013,52(6):771-775. DOI: 10.1053/j.jfas.2013.04.003.

4. Mathew P G, Sponer P, Pavlata J, et al. Our experience with double metatarsal osteotomy in the treatment of hallux valgus.[J]. Acta medica (Hradec Kralove), 2012,55(1):37-41. DOI: 10.14712/18059694.2015.73.

5. Al-Nammari S S, Christofi T, Clark C. Double First Metatarsal and Akin Osteotomy for Severe Hallux Valgus.[J]. Foot \& ankle international, 2015,36(10):1215-1222. DOI: 10.1177/1071100715589173.

6. Coughlin M J, Jones C P. Hallux valgus: demographics, etiology, and radiographic assessment[J]. Foot Ankle Int, 2007,28(7):759-777. DOI: 10.3113/FAI.2007.0759.

7. Li Y, Chen W, Tao X, et al. [Clinical effect of metatarsal diaphysis Z-shaped rotating osteotomy in moderate and severe hallux valgus with metatarsophalangeal joint incongruency][J]. Zhonghua Yi Xue Za Zhi, 2020,100(31):2423-2428. DOI: 10.3760/cma.j.cn112137-20200114-00093.

8. Fuhrmann R A, Zollinger-Kies $\mathrm{H}$, Kundert $\mathrm{H}$. Mid-term results of Scarf osteotomy in hallux valgus[J]. International Orthopaedics, 2010,34(7):981-989. DOI: 10.1007/s00264-010-0958-z.

9. Robinson A H, Cullen N P, Chhaya N C, et al. Variation of the distal metatarsal articular angle with axial rotation and inclination of the first metatarsal[J]. Foot Ankle Int, 2006,27(12):1036-1040. DOI: 10.1177/107110070602701207.

10. Park C H, Lee W C. Is Double Metatarsal Osteotomy Superior to Proximal Chevron Osteotomy in Treatment of Hallux Valgus With Increased Distal Metatarsal Articular Angle?[J]. J Foot Ankle Surg, 2018,57(2):241-246. 
DOI: $10.1053 /$ j.jfas.2017.08.020.

11. Wang X, Wen Q, Li Y, et al. Introduction the revolving scarf osteotomy for treating severe hallux valgus with an increased distal metatarsal articular angle: a retrospective cohort study[J]. BMC musculoskeletal disorders, 2019,20(1):508. DOI: 10.1186/s12891-019-2874-8.

12. Seo J, Ahn J, Boedijono D. Point-Connecting Measurements of the Hallux Valgus Deformity: A New Measurement and Its Clinical Application[J]. Yonsei Medical Journal, 2016,57(3):741. DOI: 10.3349/ymj.2016.57.3.741.

13. Frumberg D B, Naziri Q, Pivec R, et al. Rotational Deformity of the First Ray Precludes Accurate Distal Metatarsal Articular Angle Measurement in Hallux Valgus.[J]. Journal of long-term effects of medical implants, 2018,28(1):41-46. DOI: 10.1615/JLongTermEffMedlmplants.2017020615.

14. Coughlin M J. Hallux valgus in men: effect of the distal metatarsal articular angle on hallux valgus correction[J]. Foot Ankle Int, 1997,18(8):463-470. DOI: 10.1177/107110079701800802.

\section{Figures}




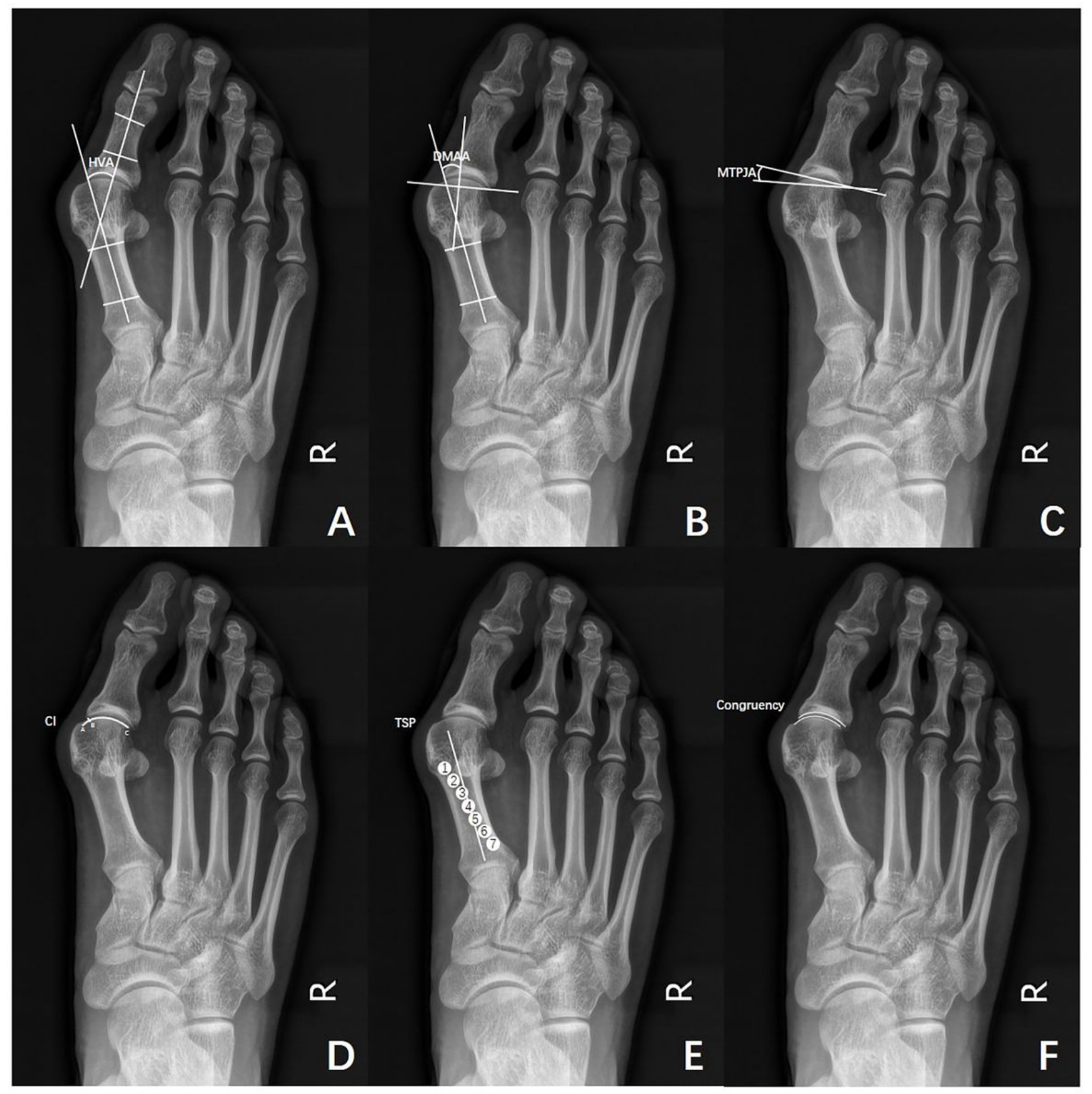

\section{Figure 1}

The imaging indicators of the first MTP joint on the weightbearing foot anterior-posterior images. A: Hallux valgus angle, HVA; B: Distal metatarsal articular angle, DMAA; C: Metatarsophalangeal joint angle, MTPJA; D: Congruency index, Cl; E: Tibial sesamoid position, TSP; F: Evaluation of the congruency of the first MTP joint. 


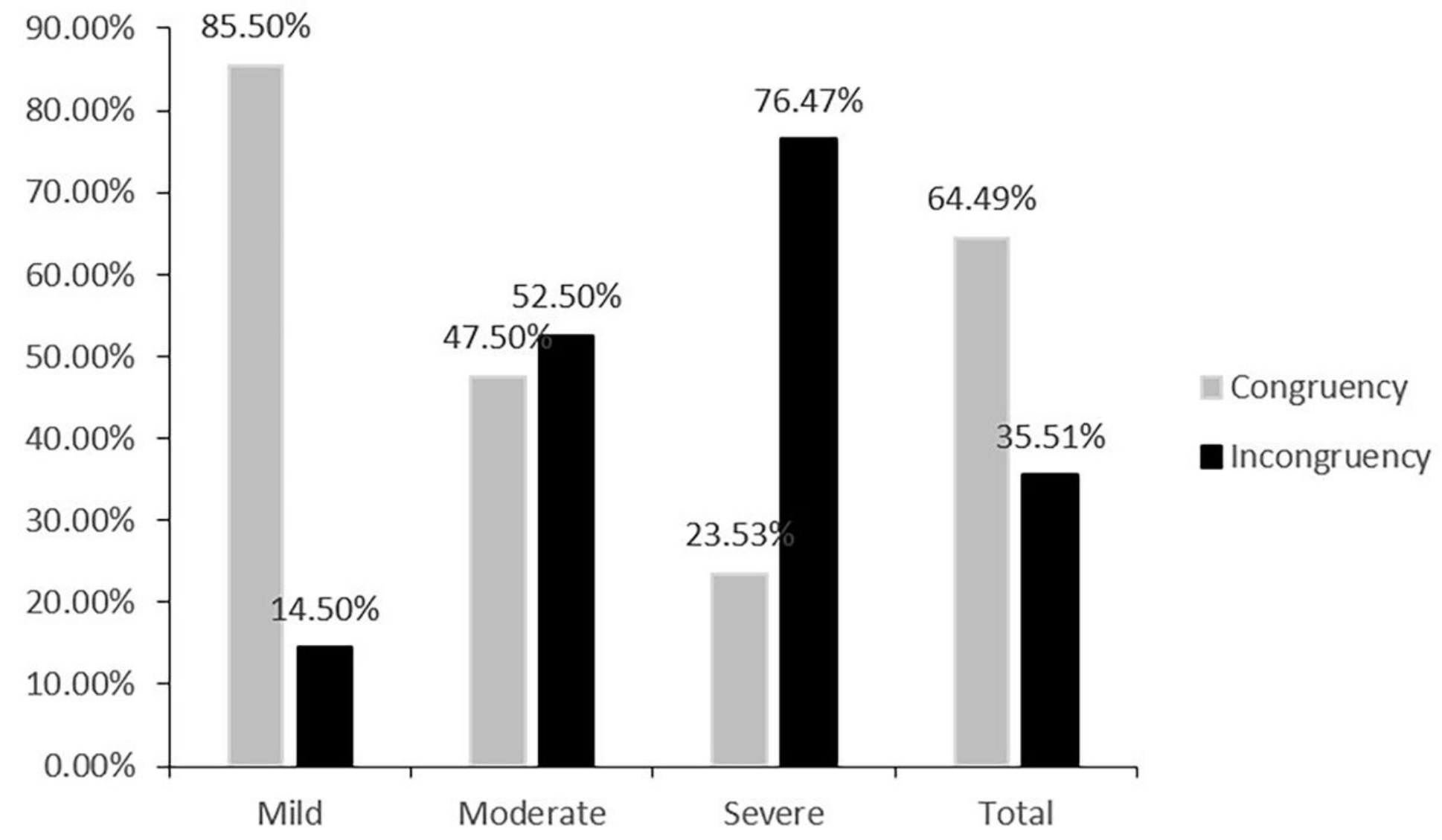

Figure 2

Distribution of congruency of the first metatarsophalangeal joint in patients with mild to severe hallux valgus 


\section{ROC curve}

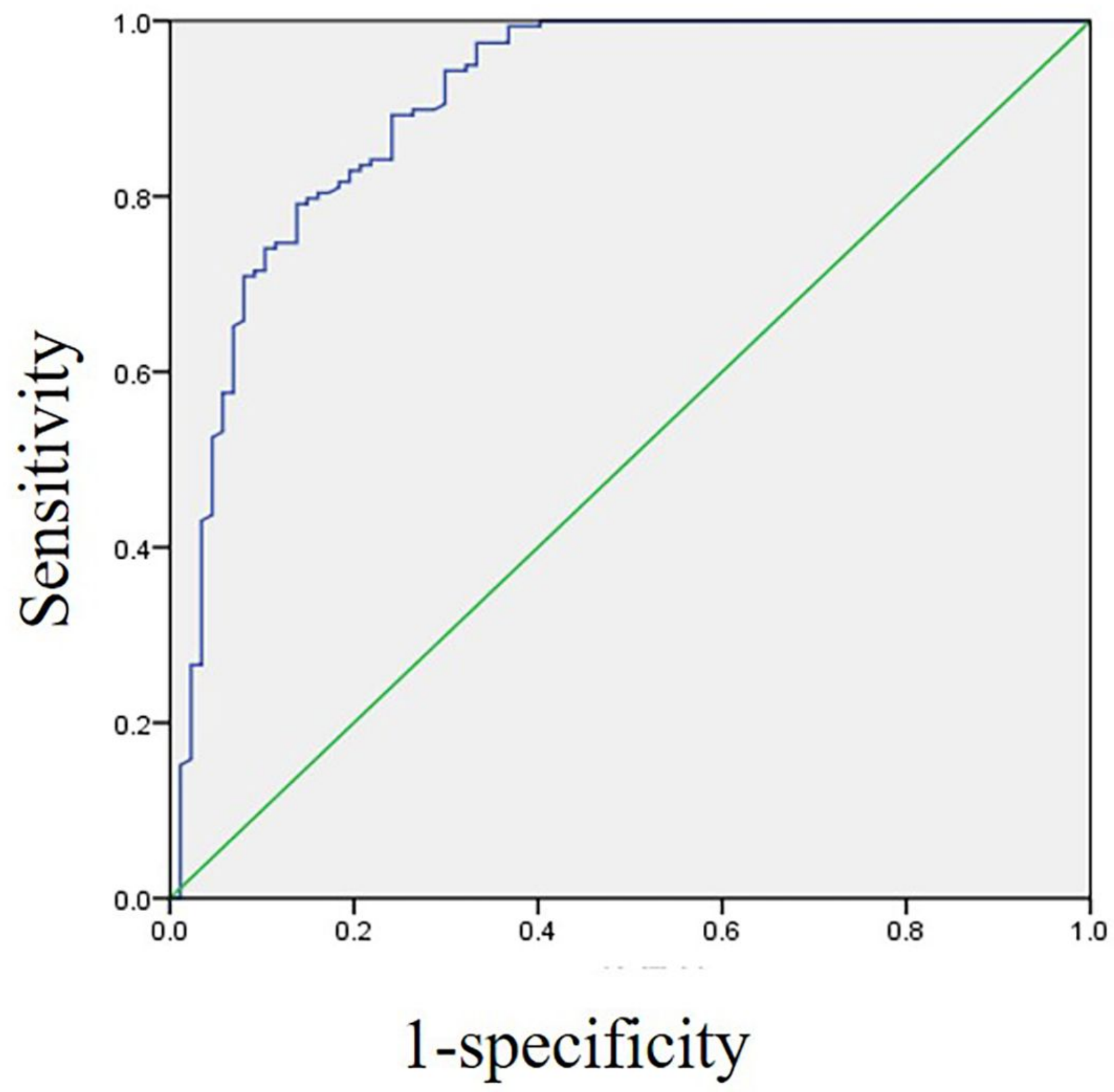

Figure 3

The ROC curve of the metatarsophalangeal joint angle (MTPJA) and congruency of the first metatarsophalangeal (MTP) joint. The area under the curve (AUC) was 0.906. 


\section{ROC curve}

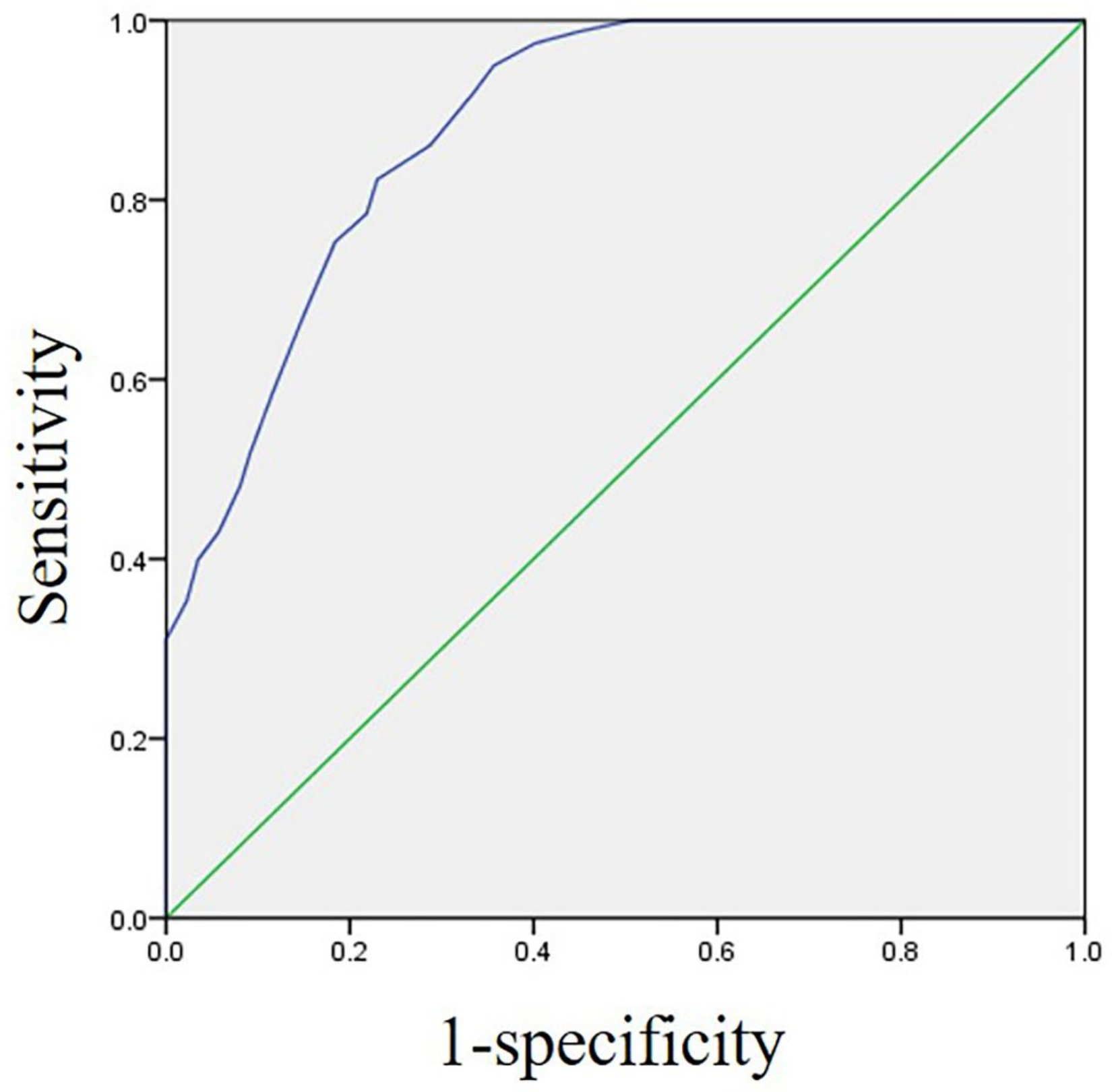

Figure 4

The ROC curve of the congruency index $(\mathrm{Cl})$ and congruency of the first metatarsophalangeal (MTP) joint. The area under the curve (AUC) was 0.884 . 